\title{
Variational Bounds to Eigenvalues of Self-Adjoint Eigenvalue Problems with Arbitrary Spectrum
}

\author{
S. Zimmermann and U. Mertins
}

\begin{abstract}
In the present paper a method by Lehmann-Maehly and Goerisch is extended to self-adjoint eigenvalue problems with arbitrary essential spectrum. This extension is obtained by consequently making use of the local character of the method. In this way, upper and lower bounds to all isolated eigenvalues are derived. In our proofs, the close relationship to Wielandt's inverse iteration becomes quite obvious.
\end{abstract}

Keywords: Eigenvalue problems, variational methods, upper and lower bounds to eigenvalues AMS subject classification: $49 \mathrm{R} 05,65$ N 25, 65 N 30, 35 P 15

\section{Introduction}

The characterization of eigenvalues of self-adjoint eigenvalue problems by a minimummaximum principle for the Rayleigh quotient forms the basis for the famous RayleighRitz method. As is well known, the method allows for a straightforward and efficient computation of non-increasing upper bounds to eigenvalues below the essential spectrum. In [14] and [15], Lehmann and Maehly independently developed complementary eigenvalue characterizations that provide a possibility to calculate corresponding lower bounds (see also [21: Chapter 4.11]). More precisely, they introduce a spectral parameter and construct minimum-maximum as well as maximum-minimum principles for Temple's quotient. From this, upper and lower bounds are deduced. In particular, the Rayleigh-Ritz method can be interpreted as a special application of the LehmannMaehly method. In general, however, the Lehmann-Maehly method is applicable only if certain quantities can be determined explicitly. Especially when treating partial differential equations, difficulties may arise. Of great importance for practical applications is therefore a generalized version by Goerisch (see $[5,8,10]$ ) that combines high flexibility and good convergence properties. Usually, very satisfying inclusion intervals are obtained from a combination of Rayleigh-Ritz and Goerisch calculations (see [2 - 6, 10, 11)).

Some very interesting eigenvalue problems however possess isolated eigenvalues $\lambda \epsilon$ $\left(\rho^{-}, \rho^{+}\right)$, where both $\rho^{-}$and $\rho^{+}$belong to the essential spectrum. Examples are the

S. Zimmermann: Techn. Univ. Clausthal, Inst. Math., Erzstr. 1, D - 38678 Clausthal-Zellerfeld

U. Mertins: Techn. Univ. Clausthal, Inst. Math., Erzstr. 1, D - Clausthal-Zellerfeld

ISSN 0232-2064 / S 2.50 C Heldermann Verlag 
Dirac equation (see [16: p. 952] and [20]), the Schrödinger operator [1] and the linearized equations of ideal magnetohydrodynamics (see [12: Chapters 1.2 and 3.2] and [17]).

So far, no bounds have been constructed for such interior eigenvalues $\lambda$. A RayleighRitz calculation does not necessarily give bounds. Moreover, it was shown that an additional convergence criterium has to be satisfied in order to guarantee convergence of Rayleigh-Ritz approximations. [7, 18]. In problems with interior eigenvalues, a spectral parameter appears to be particularly useful. We shall derive here an extension of the Goerisch method that gives bounds also to interior eigenvalues. In our development we generalize the Lehmann-Maehly variational principle in such a way that an appropriate discretization readily produces Goerisch's method - a result which in itself is at least of theoretical interest.

In Section 1, we characterize eigenvalues of a self-adjoint operator by new variational principles. In what follows, we consider two types of variationally posed eigenvalue problems as there exist two non-equivalent versions of the Goerisch method. In Section 2, we apply results of Section 1 to left-definite eigenvalue problems, Section 3 giving a numerical example for the inclusion of interior eigenvalues. In Section 4 , the rightdefinite theory is developed and applied.

\section{Characterizations for eigenvalues of self-adjoint operators}

Let $\mathbf{H}$ be a separable, complex Hilbert space with inner product $\langle\cdot, \cdot\rangle$ and norm $\|\cdot\|$, and suppose we are given a self-adjoint operator $A$ in $\mathbf{H}$ with domain $\mathcal{D}(A)$. Denote by $\sigma(A)$ and $\sigma_{e}(A)$ the spectrum and the essential spectrum of $A$,

$$
\begin{aligned}
\sigma_{e}(A)= & \{\lambda \in \sigma(A): \lambda \text { is an accumulation point of } \sigma(A)\} \\
& \cup\{\lambda \in \sigma(A): \lambda \text { is an eigenvalue of infinite multiplicity }\}
\end{aligned}
$$

If the spectrum $\sigma(A)$ of $A$ is bounded from below and begins with isolated eigenvalues of finite multiplicity,

$$
\lambda_{i}^{-} \leq \lambda_{2}^{-} \leq \ldots<\inf \sigma_{e}(A)
$$

(eigenvalues are always repeated according to their multiplicities), these eigenvalues can be characterized by well known variational principles, e.g. the Poincaré principle (cf. [22: Chapter 2])

$$
\lambda_{i}^{-}=\inf _{\substack{v \subset \mathcal{D}(\mathcal{A}) \\ \operatorname{dim} V=i}} \max _{\substack{0 \neq v \in V\\}} \frac{\langle A v, v\rangle}{\langle v, v\rangle}
$$

If in addition $\sigma_{e}(A) \neq \emptyset$, the number of isolated eigenvalues $\lambda_{i}^{-}$below $\sigma_{e}(A)$ is either infinite with $\lim _{i \rightarrow \infty} \lambda_{i}^{-}=\inf \sigma_{e}(A)$ or finite, i.e. equal to $k$ for some $k \in \mathbb{N}$. We then set $\lambda_{k+1}^{-}=\lambda_{k+2}^{-}=\ldots=\inf \sigma_{e}(A)$. As a consequence, (1.1) holds for $i \in N$ in both cases (cf. [21: Chapter 3.5]).

If $\sigma(A)$ is bounded from above and begins with isolated eigenvalues $\lambda_{i}^{+}, \sup \sigma_{e}(A)<$ $\ldots \leq \lambda_{2}^{+} \leq \lambda_{1}^{+}$, an analogous principle is obtained, with "inf" and "max" being replaced by "sup" and "min", respectively. 
Evidently, interior eigenvalues are not accessible for this kind of variational principles. Therefore, a spectral parameter $\rho$ is introduced. For fixed $\rho \in \mathbb{R}$ set

$$
\rho^{-}=\sup \left\{\lambda \in \sigma_{e}(A): \lambda<\rho\right\} \in \mathbb{R} \cup\{-\infty\} .
$$

Provided $\rho^{-}<\rho$ and $\rho$ is no accumulation point of $\left(\rho^{-}, \rho\right) \cap \sigma(A)$, this intersection is either empty or consists of a countable number of eigenvalues of finite multiplicity. It is then possible to introduce the following local notation for these eigenvalues:

$$
\rho^{-}<\ldots \leq \lambda_{-k_{\rho}^{-}}^{\rho} \leq \cdots \leq \lambda_{-2}^{\rho} \leq \lambda_{-1}^{\rho}<\rho
$$

where $k_{\rho}^{-} \in \mathbb{N}_{0}$ denotes the number of eigenvalues we are interested in.

The definition of $\rho^{+} \in \mathbb{R} \cup\{\infty\}$ and $\lambda_{+i}^{\rho}$ for $i=1, \ldots, k_{\rho}^{+}$is analogous and can thus be omitted.

If $\rho \in \mathbb{R}$ is no accumulation point of $\sigma(A)$ - though it may possibly be an eigenvalue of arbitrary multiplicity of $A$ - we have the following situation:

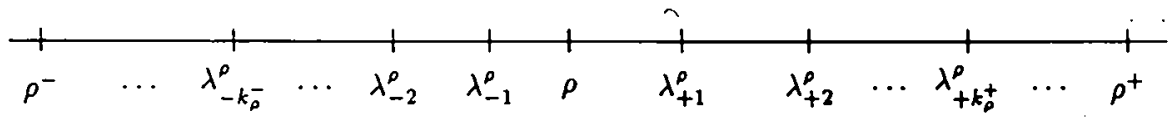

In applications the boundary points in $\mathbb{R}$ of the essential spectrum are often well known. They represent particularly well suited choices for our spectral parameter $\rho$ (see the examples of Sections 3 and 4 ).

First, we shall characterize the eigenvalues $\lambda_{ \pm i}^{\rho}$ by three different variational principles which allow to construct lower bounds to $\lambda_{-i}^{\rho}$ for $i=1, \ldots, k_{\rho}^{-}$, and upper bounds to $\lambda_{+i}^{\rho}$ for $i=1, \ldots, k_{\rho}^{+}$. By an appropriate choice for $\rho$ it is thus possible to reach every isolated eigenvalue of $A$.

In order to construct variational principles for $\lambda_{ \pm i}^{\rho}$ let

$$
D_{\rho}=\{u \in \mathcal{D}(A): A u=\rho u\}
$$

and consider the restriction of $A-\rho I$ to

$$
\mathcal{D}(A) \Theta D_{\rho}=\left\{u \in \mathcal{D}(A):\langle u, v\rangle=0 \text { for all } v \in D_{\rho}\right\}
$$

As $D_{\rho}$ is a reducing subspace for $A$, we have the inclusion

$$
\sigma(A) \subset \sigma\left(A_{\mid \mathcal{D}(A) \Theta D_{\rho}}\right) \cup\{\rho\} .
$$

In particular, $(A-\rho I)_{\mid \mathcal{D}(A) \ominus D \text {, is invertible. We set }}$

$$
R_{\rho}=\left((A-\rho I)_{\left.\mid \mathcal{D}(A) \ominus D_{\rho}\right)^{-1}}\right.
$$

and consider the eigenvalue problem for the self-adjoint operator $R_{\rho}$ in $D_{\rho}^{\perp}$. Now, $(\lambda, u) \in \mathbb{R} \times \mathcal{D}(A)$ with $\lambda \neq \rho$ is an eigenpair of $A$ if and only if $\left((\lambda-\rho)^{-1}, u\right)$ is an eigenpair of $R_{\rho}$. Set

$$
\sigma_{i}^{-}=\left(\lambda_{-i}^{\rho}-\rho\right)^{-1} \quad \text { and } \quad \sigma_{i}^{+}=\left(\lambda_{+i}^{\rho}-\rho\right)^{-1}
$$


for $i=1, \ldots, k_{\rho}^{\mp}$, so that we arrive at

$$
\lambda_{-i}^{\rho}=\rho+\left(\sigma_{i}^{-}\right)^{-1} \quad \text { and } \quad \lambda_{+i}^{\rho}=\rho+\left(\sigma_{i}^{+}\right)^{-1}
$$

respectively.

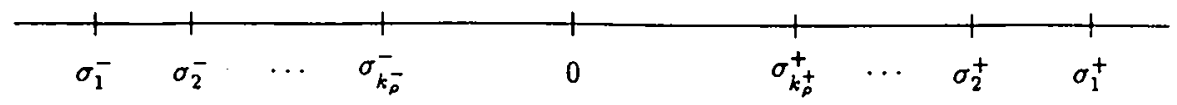

Poincaré's principle applied to $R_{\rho}$ now gives the following characterizations.

Theorem 1.1: Let $\rho^{-}<\rho$. Then

$$
\lambda_{-i}^{\rho}=\rho+\left(\inf _{V} \max _{0 \neq v \in V} \frac{\langle A v, v\rangle-\rho(v, v\rangle}{\|(A-\rho I) v\|^{2}}\right)^{-1}
$$

holds for $i=1, \ldots, k_{\rho}^{-}$, the infimum being taken over all subspaces $V$ of $\mathcal{D}(A)$ that satisfy

$$
\operatorname{dim} V=i \quad \text { and } \quad A v \neq \rho v \text { for all } 0 \neq v \in V .
$$

If $\rho<\rho^{+}$, we have

$$
\lambda_{+i}^{\rho}=\rho+\left(\sup _{V} \min _{0 \neq v \in V} \frac{\langle A v, v\rangle-\rho\langle v, v\rangle}{\|(A-\rho I) v\|^{2}}\right)^{-1}
$$

for $i=1, \ldots, k_{\rho}^{+}$and with $V$ as in (1.4).

Proof: Suppose $k_{\rho}^{-} \geq 1$. The spectral mapping theorem [13: Chapter III, Section 6.3] gives

$$
\tilde{\sigma}\left(R_{\rho}\right)=\left(\tilde{\sigma}\left(A_{\mid \mathcal{D}(A) \Theta D_{\rho}}\right)-\rho\right)^{-1}
$$

where

$$
\tilde{\sigma}(S)= \begin{cases}\sigma(S) & \text { if } S \text { is bounded } \\ \sigma(S) \cup\{\infty\} & \text { otherwise. }\end{cases}
$$

The mapping $\lambda \longmapsto \sigma=(\lambda-\rho)^{-1}$ transforms the eigenvalues $\lambda_{-i}^{\rho}\left(i=1, \ldots, k_{\rho}^{-}\right)$of $A$ into eigenvalues $\sigma_{i}^{-}$of $R_{\rho}$ without changing their multiplicities. The spectrum of $R_{\rho}$ is bounded from below and begins with the eigenvalues

$$
\sigma_{1}^{-} \leq \ldots \leq \sigma_{k_{-}^{-}}^{-}<\inf \sigma_{e}\left(R_{p}\right) .
$$

We apply Poincaré's principle (1.1),

$$
\sigma_{i}^{-}=\inf _{\substack{u \in D\left(R_{\rho}\right) \\ \operatorname{dim} U=i}} \max _{0 \neq u \in U} \frac{\left\langle R_{\rho} u, u\right\rangle}{\langle u, u\rangle} \quad \text { for } i=1, \ldots, k_{\rho}^{-},
$$

substitute $w=R_{\rho} u$ and replace $U \subset \mathcal{D}\left(R_{\rho}\right)$ by $W \subset \mathcal{D}(A) \ominus D_{\rho}$,

$$
\sigma_{i}^{-}=\inf _{\substack{w \subset \mathcal{D}(A) \Theta D, \operatorname{dim} w=i}} \max _{0 \neq w \in W} \frac{\langle w,(A-\rho I) w\rangle}{\|(A-\rho I) w\|^{2}} \quad \text { for } i=1, \ldots, k_{\rho}^{-} .
$$


Evidently, every subspace $W$ admissible in (1.7) satisfies (1.4). Conversely, suppose that (1.4) holds for a subspace $V$ of $\mathcal{D}(A)$, and denote by $P$ the orthogonal projector in $\mathbf{H}$ onto the orthogonal complement of $D_{\rho}$. Then $W=P V$ is admissible in (1.7), and for $v \in V$ and $w=P v \in W$ we have

$$
(A-\rho I) v=(A-\rho I) w \quad \text { and } \quad\langle v,(A-\rho I) v\rangle=\langle w,(A-\rho I) w\rangle .
$$

Thus, we find

$$
\sigma_{i}^{-}=\inf _{\substack{c \mathcal{D}(1) \\(1.4)}} \max _{0 \neq v \in V} \frac{\langle v,(A-\rho I) v\rangle}{\|(A-\rho I) v\|^{2}} \quad \text { for } i=1, \ldots, k_{\rho}^{-}
$$

giving (1.3). Formula (1.5) is obtained in exactly the same way

Note that equation (1.6) together with (1.2) already indicates a close relationship to Wielandt's inverse iteration dealing with matrix problems.

Motivated by Goerisch's approach we now generalize the characterisations (1.3) and (1.5) of the eigenvalues in two different ways, in order to enlarge the range of applicability of the derived methods (cf. Remark 2.2).

In addition to our initial assumptions suppose that $\mathbf{X}$ is a complex Hilbert space with inner product $s(\cdot, \cdot)$, and that $T: \mathbf{H} \longrightarrow \mathbf{X}$ is an isometry

$$
s(T u, T v)=\langle u, v\rangle \quad \text { for } \quad u, v \in \mathbf{H} .
$$

The choice

$$
\mathbf{X}=\mathbf{H}, \quad s(u, v)=\langle u, v\rangle \quad \text { for } u, v \in \mathbf{H}, \quad T v=v \text { for } v \in \mathbf{H}
$$

gives the procedure of Lehmann-Maehly (cf. Remark 2.2).

Denote by

$$
\mathbf{X}^{\circ}=\{w \in \mathbf{X}: s(w, T u)=0 \text { for } u \in \mathbf{H}\} .
$$

the orthogonal complement of $T \mathbf{H}$ in $\mathbf{X}$. The quantities $\mathbf{X}, s$ and $T$ provide the following complementary variational principles.

Lemma 1.2: Suppose $S$ is a self-adjoint operator in $\mathbf{H}$ with domain $\mathcal{D}(S)$. Then for $v \in \mathcal{D}(S)$ given, we have

$$
\begin{aligned}
& \langle S v, S v\rangle=\max \{\langle S v, f\rangle+\overline{\langle S v, f\rangle}-\langle f, f\rangle: f \in \mathbf{H}\} \\
& \langle S v, S v\rangle=\min \left\{s\left(T S v+w^{\circ}, T S v+w^{\circ}\right): w^{\circ} \in \mathbf{X}^{\circ}\right\}
\end{aligned}
$$

Proof: For $v \in \mathcal{D}(S), f \in \mathbf{H}$ and $z=T S v+w^{\circ}$ with $w^{\circ} \in \mathbf{X}^{\circ}$ one obtains

$$
0 \leq\langle S v-f, S v-f\rangle=\langle S v, S v\rangle-\langle S v, f\rangle-\overline{\langle S v, f\rangle}+\langle f, f\rangle
$$

and

$$
0 \leq s(z-T S v, z-T S v)=s(z, z)-\langle S v, S v\rangle
$$

which gives the assertion 
We mention that relation (1.9) still holds when $s(\cdot, \cdot)$ denotes a positive semidefinite sesquilinear form in $\mathbf{X}$. For simplicity, we restrict our considerations to the positive definite case. It was pointed out in [9] that many well known complementary variational principles can be derived from (1.9) by an appropriate choice of $\mathbf{X}, s(\cdot, \cdot)$ and $T$, e.g. those given in [19: Chapter 4.1.4].

New characterizations for $\lambda_{ \pm i}^{\rho}$ can be deduced by application of (1.9) with $S=A$ and $S=A^{-1}$, respectively.

Theorem 1.3: Let $\rho^{-}<\rho$. Then,

$$
\lambda_{-i}^{\rho}=\rho+\left(\inf _{\substack{\mathcal{C}(1) \\(1,4)}} \max _{0 \neq v \in V} \min _{w \in T A v+\mathrm{X}} \frac{\langle A v, v\rangle-\rho\langle v, v\rangle}{s(w, w)-2 \rho\langle A v, v\rangle+\rho^{2}\langle v, v\rangle}\right)^{-1}
$$

holds for $i=1, \ldots, k_{\rho}^{-}$.

Proof: Let $1 \leq i \leq k_{\rho}^{-}$. We apply Theorem 1.1 and relation (1.9) for $S=A$,

$$
\sigma_{i}^{-}=\inf _{\substack{v \in \mathcal{D}(1) \\(1.4)}} \max _{0 \neq v \in V} \frac{\langle A v, v\rangle-\rho\langle v, v\rangle}{\min _{w \in T A v+X^{\circ}}\{s(w, w)\}-2 \rho\langle A v, v\rangle+\rho^{2}\langle v, v\rangle} .
$$

Due to $\sigma_{i}^{-}<0$ it suffices to take the infimum over subspaces $V$ of $\mathcal{D}(A)$ satisfying

$$
\operatorname{dim} V=i \quad \text { and } \quad\langle A v, v\rangle<\rho\langle v, v\rangle \text { for all } 0 \neq v \in V .
$$

Inserting this into (1.11) provides

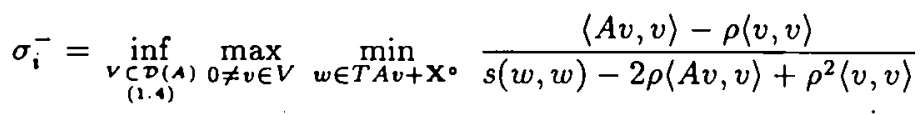

and thus (1.10)

Theorem 1.4: Suppose $0 \notin \sigma(A), \rho>0$ and $\rho^{-}<\rho$. Then we have

$$
\lambda_{-i}^{\dot{\rho}}=\rho+\rho\left(\inf _{V} \max _{0 \neq v \in V} \min _{w \in T A^{-1} v+X^{\circ}} \frac{\langle v, v\rangle-\rho\left\langle A^{-1} v, v\right\rangle}{\langle v, v\rangle-2 \rho\left\langle A^{-1} v, v\right\rangle+\rho^{2} s(w, w)}-1\right)^{-1}
$$

for $1 \leq i \leq k_{\rho}^{-}$such that $\lambda_{-i}^{\rho}$ is positive. Here, $V$ varies over subspaces of $\mathbf{H}$ satisfying

$$
\operatorname{dim} V=i \quad \text { and } \quad v \neq \rho A^{-1} v \text { for all } 0 \neq v \in V \text {. }
$$

Proof: Due to our assumptions, $A: \mathcal{D}(A) \longrightarrow \mathbf{H}$ is a bijection. We combine Theorem 1.1 and relation (1.9) for $S=A^{-1}$, giving

$$
\begin{aligned}
& \sigma_{i}^{-}=\inf _{\substack{c \mathcal{D}(\Lambda) \\
(1.4)}} \max _{0 \neq v \in V} \frac{\langle A v, v\rangle-\rho\langle v, v\rangle}{\langle A v, A v\rangle-2 \rho\langle A v, v\rangle+\rho^{2}\langle v, v\rangle} \\
& =\inf _{\substack{v \subset \mathbb{C} \\
(1.14)}} \max _{0 \neq v \in V} \frac{\left\langle v, A^{-1} v\right\rangle-\rho\left\langle A^{-1} v, A^{-1} v\right\rangle}{\langle v, v\rangle-2 \rho\left\langle v, A^{-1} v\right\rangle+\rho^{2}\left\langle A^{-1} v, A^{-1} v\right\rangle}
\end{aligned}
$$

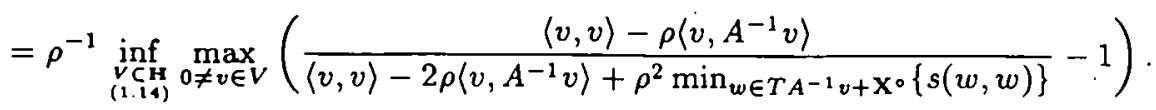


Here, $\lambda_{-i}^{\rho}>0$ implies $\sigma_{i}^{-}<-\rho^{-1}<0$. Thus, we can restrict the infimum to subspaces $V$ of $\mathbf{H}$ satisfying

$$
\operatorname{dim} V=i \quad \text { and } \quad\langle v, v\rangle<\rho\left\langle v, A^{-1} v\right\rangle \text {. for all } 0 \neq v \in V .
$$

Employing (1.15) we find

$$
\sigma_{i}^{-}=\frac{1}{\rho}\left(\inf _{\substack{V \subset \mathbb{v} \\(1,14)}} \max _{0 \neq v \in V} \min _{w \in T A^{-1} v+\mathrm{X}^{\circ}} \frac{\langle v, v\rangle-\rho\left\langle v, A^{-1} v\right\rangle}{\langle v, v\rangle-2 \rho\left\langle v, A^{-1} v\right\rangle+\rho^{2} s(w, w)}-1\right)
$$

and the assertion is proved

Through a reformulation of Theorems 1.3 and 1.4 one can derive characterizations for $\lambda_{-i}^{\rho}$ that involve the following generalized Temple quotients:

$$
\begin{array}{cl}
\frac{s(w, w)-\rho\langle A v, v\rangle}{\langle A v, v\rangle-\rho\langle v, v\rangle} & \text { for } v \in \mathcal{D}(A), w \in T A v+\mathbf{X}^{\circ},\langle v, A v\rangle-\rho\langle v, v\rangle \neq 0 \\
\frac{\langle v, v\rangle-\rho\left\langle v, A^{-1} v\right\rangle}{\left\langle v, A^{-1} v\right\rangle-\rho s(w, w)} & \text { for } v \in \mathbf{H}, w \in T A^{-1} v+\mathbf{X}^{\circ},\left\langle v, A^{-1} v\right\rangle-\rho s(w, w) \neq 0
\end{array}
$$

In the situation of (1.8), these yield the classical Temple quotients

$$
\frac{\langle A v,(A-\rho I) v\rangle}{\langle v,(A-\rho I) v\rangle} \quad \text { and } \quad \frac{\left\langle v,\left(I-\rho A^{-1}\right) v\right\rangle}{\left\langle A^{-1} v,\left(I-\rho A^{-1}\right) v\right\rangle} .
$$

Observe that by $\rho \longrightarrow \pm \infty$ in (1.16) and $\rho \longrightarrow 0$ in (1.17) the Rayleigh quotient of $A$ and the inverse of the Rayleigh quotient of $A^{-1}$ is produced.

Corollary 1.5: Let $\rho^{-}<\rho$. Then

$$
\lambda_{-i}^{\rho}=\sup _{\substack{v<D(1) \\(1,12)}} \min _{0 \neq v \in V} \max _{w \in T A v+X^{\circ}} \frac{s(w, w)-\rho\langle A v, v\rangle}{\langle A v ; v\rangle-\rho\langle v, v\rangle}
$$

holds for $i=1, \ldots, k_{\rho}^{-}$.

Corollary 1.6: Suppose $0 \notin \sigma(A), \rho>0$ and $\rho^{-}<\rho$.Then

$$
\lambda_{-i}^{\rho}=\sup _{\substack{V \subset H \\(1,1 b)}} \min _{0 \neq v \in V} \max _{w \in T A^{-1} v+\mathbf{X}} \cdots \frac{\langle v, v\rangle-\rho\left(v, A^{-1} v\right)}{\left\langle v, A^{-1} v\right)-\rho s(w, w)}
$$

holds for $1 \leq i \leq k_{\rho}^{-}$such that $\lambda_{-i}^{\rho}$ is positive.

Remark 1.7: In Theorems 1.3 and 1.4, interchanging "inf" and "sup" as well as "min" and "max" gives characterizations for $\lambda_{+i}^{\rho}$, with (1.12) and (1.15) being replaced by

$$
\operatorname{dim} V=i \quad \text { and } \quad\langle A v, v\rangle>\rho\langle v, v\rangle \text { for all } 0 \neq v \in V
$$


and

$$
\operatorname{dim} V=i \quad \text { and } \quad\left\langle v, A^{-1} v\right\rangle>\rho\left\langle A^{-1} v, A^{-1} v\right\rangle \text { for all } 0 \neq v \in V,
$$

respectively. The same applies to Corollaries 1.5 and 1.6. In Corollary 1.6, however, $w \in T A v+\mathbf{X}^{\circ}$ is then further restricted by

$$
\left\langle v, A^{-1} v\right\rangle>\rho s(w, w)
$$

The proofs follow the exact pattern of the previous ones.

In applications one often finds eigenvalue problems which are given in variational form. In what follows we shall therefore employ the results of this section in order to construct variational bounds to eigenvalues of such problems.

\section{The left-definite Goerisch method}

Let $\mathbf{H}_{a}$ be a separable, complex Hilbert space with inner product $a(\cdot, \cdot)$ and norm $\|\cdot\|$. Suppose $b(\cdot, \cdot)$ is a continuous, Hermitian, sesquilinear form on $\mathbf{H}_{a} \times \mathbf{H}_{a}$ such that $u \in \mathbf{H}_{a}$ and $b(u, v)=0$ for all $v \in \mathbf{H}_{a}$ implies $u=0$. We then consider the left-definite, variationally posed eigenvalue problem

$$
\left.\begin{array}{l}
\text { Find } \lambda \in \mathbb{R} \text { and non-trivial } u \in \mathbf{H}_{a} \text { such } \\
\text { that } a(u, v)=\lambda b(u, v) \text { holds for all } v \in \mathbf{H}_{a} .
\end{array}\right\}
$$

Denote by $B$ the bounded self-adjoint operator in $\mathbf{H}_{\boldsymbol{a}}$ that satisfies

$$
a(B u, v)=b(u, v) \quad \text { for all } u, v \in \mathbf{H}_{\mathbf{a}} .
$$

By assumption, $B$ possesses a self-adjoint inverse $B^{-1}$ in $\mathbf{H}_{\mathbf{a}}$. For an application of the results of Section 1, we make the identifications

$$
\mathbf{H}=\mathbf{H}_{a}, \quad\langle u, v\rangle=a(u, v) \quad \text { for all } u, v \in \mathbf{H}_{a}, \quad A=B^{-1}
$$

and we assume that $\mathbf{X}$ is a complex Hilbert space with inner product $s(\cdot, \cdot)$ and equipped with an isometry $T: \mathbf{H}_{\boldsymbol{a}} \longrightarrow \mathbf{X}$. Fix $\rho \in \mathbb{R}, \rho>0$. All assumptions of Section 1 are then satisfied with

$$
D_{\rho}=\left\{u \in \mathbf{H}_{a}: a(u, v)=\rho b(u, v) \text { for all } v \in \mathbf{H}_{a}\right\}
$$

Evidently, (2.1) is equivalent to the eigenvalue problem for $A$. Hence, $\sigma(A)$ and $\sigma_{e}(A)$ represent the spectrum and the essential spectrum of $(2.1)$, with $0 \notin \sigma(A)$. We adopt from Section 1 the definition of $\rho^{ \pm}, k_{\rho}^{ \pm}$and $\lambda_{ \pm i}^{\rho}$.

In Theorem 1.4 and Corollary 1.6, we have formulated two equivalent characterizations for $\lambda_{-i}^{\rho}$ each of which is easily discretized. A discretization of (1.18) has the advantage that no transformation of eigenvalues is needed. Furthermore, $\rho \longrightarrow 0$ obviously produces the Rayleigh-Ritz method. In general, however, the finite-dimensional eigenvalue problem derived from (1.18) involves two indefinite matrices. For this reason we prefer (1.13) which produces right-definite matrix eigenvalue problems in any case. We thus continue with a reformulation of Theorem 1.4 for left-definite, variationally posed eigenvalue problems. 
Corollary 2.1: Let $\rho^{-}<\rho$. Then

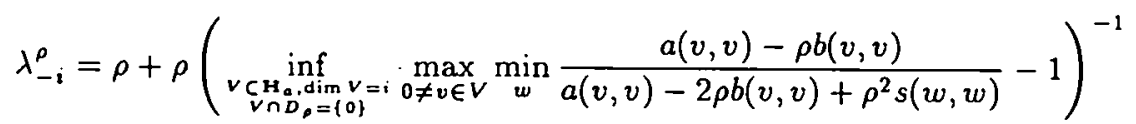

holds for $1 \leq i \leq k_{\rho}^{-}$such that $\lambda_{-i}^{\rho}$ is positive, the minimum being taken over $w \in \mathbf{X}$ such that

$$
s(w, T u)=b(v, u) \quad \text { for all } u \in \mathbf{H}_{\mathbf{a}} .
$$

Proof: Since (2.3) is equivalent to the inclusion $w \in T B v+\mathbf{X}^{\circ}$, the assertion follows immediately from Theorem 1.4

An analogous characterization holds for $\lambda_{+i}^{\rho}$ (see Remark 1.7).

For a discretization, suppose $n \in \mathbb{N}, m \in \mathbb{N}_{0}$, and suppose the following:

(L1) $v_{1}, \ldots, v_{n} \in \mathbf{H}_{a}$ are linearly independent.

(L2) $w_{1}^{\star}, \ldots, w_{n}^{\star} \in \mathbf{X}$ satisfy $s\left(w_{i}^{*}, T u\right)=b\left(v_{i}, u\right)$ for all $u \in \mathbf{H}_{u} \quad(i=1, \ldots, n)$.

(L3) $w_{0}^{\circ}, \ldots, w_{m}^{\circ} \in \mathbf{X}^{\circ}$, where $w_{0}^{\circ}=0$ and $w_{1}^{\circ}, \ldots, w_{m}^{\circ}$ are linearly independent.

Here $v_{1}, \ldots, v_{n}$ are Rayleigh-Ritz trial functions. In addition, the Goerisch approach requires $w_{i}^{*}$ and $w_{j}^{\circ}$ depending on $v_{i}$ and the choice of $\mathbf{X}, s$ and $T$.

Remark 2.2: In the Lehmann-Maehly approach which is characterized by (1.8), one has

$$
\mathbf{X}=\mathbf{H}_{a}, \quad s(\cdot, \cdot)=a(\cdot, \cdot), \quad T=I, \quad \mathbf{X}^{\circ}=\{0\}
$$

so that $w_{i}^{\star}$ is the unique solution of the linear problem

$$
\text { Seek } w \in \mathbf{H}_{a} \text { such that } a(w, u)=b\left(v_{i}, u\right) \text { for all } u \in \mathbf{H}_{a} \text {. }
$$

Only if this problem is too expensive or even impossible to solve, Goerisch's version is applied. It replaces (2.4) by the linear problem

$$
\text { Seek } w \in \mathbf{X} \text { such that } s(w, T u)=b\left(v_{i}, u\right) \text { for all } u \in \mathbf{H}_{\mathbf{a}} \text {. }
$$

Provided $\mathbf{X}^{\circ} \neq\{0\}$, there exists a manifold of solutions. With $X, s$ and $T$ being chosen appropriately, it is much easier to find a particular solution of problem (2.5) than to solve exactly problem (2.4). Solutions $w_{j}^{\circ} \in \mathbf{X}^{\circ}$ of the homogeneous equation (2.5) are then used to approximate the residual $T w_{i, L}^{*}-w_{i, G}^{*}$, where $w_{i, L}^{*}$ and $w_{i, G}^{*}$ denote solutions of problems (2.4) and (2.5), respectively. Note that the idea of this approach is not to improve Lehmann-Maehly bounds but to enlarge widely the range of applicability of the method.

Set

$$
V_{n}=\operatorname{span}\left\{v_{1}, \ldots, v_{n}\right\} \quad \text { and } \quad \mathbf{X}_{m}^{\circ}=\operatorname{span}\left\{w_{0}^{\circ}, \ldots, w_{m}^{\circ}\right\}
$$

For convenience, we assume

$$
V_{n} \cap D_{\rho}=\{0\}
$$


Whenever (2.6) is violated, one eliminates from $V_{n}$ all exact eigenvectors corresponding to $\rho$. Let

$$
\begin{aligned}
& a_{i k}^{(1)}=a\left(v_{k}, v_{i}\right), \quad a_{i k}^{(2)}=b\left(v_{k}, v_{i}\right), \quad a_{i k}^{(3)}=a\left(B v_{k}, B v_{i}\right) \quad(i, k=1, \ldots, n) \\
& c_{i k}^{(11)}=s\left(w_{k}^{\star}, w_{i}^{\star}\right) \quad(i, k=1, \ldots, n) \\
& c_{i k}^{(12)}=\left\{\begin{array}{ll}
s\left(w_{k}^{\circ}, w_{i}^{\star}\right) & \text { if } m>0 \\
0 & \text { if } m=0
\end{array} \quad(i=1, \ldots, n ; k=1, \ldots, \max \{1, m\})\right. \\
& c_{i k}^{(22)}=\left\{\begin{array}{ll}
s\left(w_{k}^{\circ}, w_{i}^{\circ}\right) & \text { if } m>0 \\
1 & \text { if } m=0
\end{array} \quad(i, k=1, \ldots, \max \{1, m\})\right. \\
& A_{j}=\left(a_{i k}^{(j)}\right) \quad(j=1,2,3) \\
& C_{j l}=\left(c_{i k}^{(j l)}\right) \quad(j, l=1,2 ; j \leq l) .
\end{aligned}
$$

Lemma 2.3: Let $v=\sum_{i=1}^{n} x_{i} v_{i}$ with $x=\left(x_{1}, \ldots, x_{n}\right)^{t} \in \boldsymbol{C}^{n}, w^{*}=\sum_{i=1}^{n} x_{i} w_{i}^{*}$, and $w^{\circ} \in \mathbf{X}_{m}^{\circ}$ with

$$
s\left(w^{\circ}, w\right)=-s\left(w^{\star}, w\right) \quad \text { for all } w \in \mathbf{X}_{m}^{\circ}
$$

Then

$$
\begin{aligned}
x^{H}\left(C_{11}-C_{12} C_{22}^{-1} C_{12}^{H}\right) x & =s\left(w^{\star}+w^{\circ}, w^{\star}+w^{\circ}\right) \\
& =\min \left\{s\left(w^{\star}+w, w^{\star}+w\right): w \in \mathbf{X}_{m}^{\circ}\right\} \\
& \geq x^{H} A_{3} x
\end{aligned}
$$

holds.

Proof: (i) For $m>0$, there exists $y=\left(y_{1}, \ldots, y_{m}\right)^{t} \in \boldsymbol{C}^{m}$ such that $w^{\circ}=$ $\sum_{i=1}^{m} y_{i} w_{i}^{\circ}$. The equation (2.7) is equivalent to $C_{22} y=-C_{12}^{H} x$, giving

$$
s\left(w^{\star}+w^{\circ}, w^{\star}+w^{\circ}\right)=s\left(w^{\star}+w^{\circ}, w^{\star}\right)=x^{H}\left(C_{11}-C_{12} C_{22}^{-1} C_{12}^{H}\right) x .
$$

Trivially, equality holds in (2.8) for $m=0$.

(ii) For arbitrary $w \in \mathbf{X}_{m}^{\circ}$, equation (2.7) gives

$$
s\left(w^{\star}+w, w^{\star}+w\right)=s\left(w^{\star}+w^{\circ}, w^{\star}+w^{\circ}\right)+s\left(w^{\circ}-w, w^{\circ}-w\right) .
$$

(iii) Since $w^{\star} \in T B v+\mathbf{X}^{\circ}, w^{\circ} \in \mathbf{X}_{m}^{\circ}$, Lemma 1.2 yields

$$
s\left(w^{\star}+w^{\circ}, w^{\star}+w^{\circ}\right) \geq \min \left\{s(T B v+w, T B v+w): w \in \mathbf{X}^{\circ}\right\}=a(B v, B v) .
$$

Thus the assertion is proved

Consider the matrix eigenvalue problem

$$
\tau \in \mathbb{R}, x \in \boldsymbol{C}^{n}: \quad\left(A_{1}-\rho A_{2}\right) x=\tau\left(A_{1}-2 \rho A_{2}+\rho^{2}\left(C_{11}-C_{12} C_{22}^{-1} C_{12}^{H}\right)\right) x .
$$

Assumption (2.6) guarantees the positive definiteness of $A_{1}-2 \rho A_{2}+\rho^{2} A_{3}$. By Lemma 2.3 , this implies the existence of $n$ real eigenvalues and corresponding eigenvectors for 
problem (2.9). Observe that $C_{11}-C_{12} C_{22}^{-1} C_{12}^{H}=A_{3}$ if the Lehmann-Maehly method is applied. Denote all eigenvalues $\tau$ of problem (2.9) in $\mathbb{R} \backslash[0,1]$ by $\tau_{i}^{-}$and $\tau_{i}^{+}$and arrange them in the order

$$
\tau_{1}^{-} \leq \ldots \leq \tau_{n^{-}}^{-}<0<1<\tau_{n^{+}}^{+} \leq \ldots \leq \tau_{1}^{+}
$$

with $n^{-}+n^{+} \leq n$. Then we set

$$
\begin{array}{ll}
\Lambda_{-i}^{\rho[n, m]}=\rho+\rho\left(\tau_{i}^{-}-1\right)^{-1} & \text { for } i=1, \ldots, n^{-} \\
\Lambda_{+i}^{\rho[n, m]}=\rho+\rho\left(\tau_{i}^{+}-1\right)^{-1} & \text { for } i=1, \ldots, n^{+}
\end{array}
$$

Theorem 2.4: For $\rho^{-}<\rho$ and $1 \leq i \leq \min \left\{n^{-}, k_{\rho}^{-}\right\}$, we have

$$
\Lambda_{-i}^{\rho[n, m]} \leq \lambda_{-i}^{\rho}
$$

For $\rho<\rho^{+}$and $1 \leq i \leq \min \left\{n^{+}, k_{\rho}^{+}\right\}$, we have

$$
\Lambda_{+i}^{\rho[n, m]} \geq \lambda_{+i}^{\rho}
$$

Proof: We restrict ourselfes to the proof of (2.10). With the notation introduced in Lemma 2.3, we find

$$
\begin{aligned}
0>\tau_{i}^{-} & =\min _{\substack{v \subset C^{n} \\
\operatorname{dim} V=i}} \max _{0 \neq x \in V} \frac{x^{H}\left(A_{1}-\rho A_{2}\right) x}{x^{H}\left(A_{1}-2 \rho A_{2}+\rho^{2}\left(C_{11}-C_{12} C_{22}^{-1} C_{12}^{H}\right)\right) x} \\
& =\min _{\substack{v \subset V_{n} \\
\operatorname{dim} V=i}} \max _{0 \neq v \in V} \frac{a(v, v)-\rho b(v, v)}{a(v, v)-2 \rho b(v, v)+\rho^{2} s\left(w^{\star}+w^{\circ}, w^{\star}+w^{\circ}\right)}, \\
& =\min _{\substack{V \subset V_{n} \\
d i m V=i}} \max _{0 \neq v \in V} \min _{w \in w^{*}+X_{m}^{\circ}} \frac{a(v, v)-\rho b(v, v)}{a(v, v)-2 \rho b(v, v)+\rho^{2} s(w, w)} .
\end{aligned}
$$

By construction, we have $V_{n} \subset \mathbf{H}_{a}, V_{n} \cap D_{\rho}=\{0\}$ as well as $s(w, T u)=b(v, u)$ for $u \in \mathbf{H}_{\mathrm{a}}$ and $w \in w^{\star}+\mathbf{X}_{m}^{\circ}$. Thus,

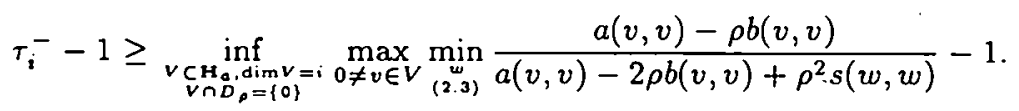

Corollary 2.1 provides 2.10

Remark 2.5: Note that the selfadjoint operator $A$ in $\mathbf{H}$ is only of theoretical interest, and that it is not required in the calculation of bounds.

For convergence results, see [24]. 


\section{Numerical example}

We shall consider an eigenvalue problem for partial differential equations that was suggested in [7] as a model problem for the study of numerical phenomina in the spectral approximation of the ideal linear magnetohydrodynamics equations ("pollution effect").

Let

$$
\Omega=(-1,1) \times(-1,1) \subset \mathbb{R}^{2} \quad \text { and } \quad \mathbf{H}_{a}=H_{0}^{1}(\Omega) \times\left(L_{2}(\Omega) \times L_{2}(\Omega)\right)
$$

We define

$$
\begin{aligned}
& a(u, v)=\int_{\Omega}\left(\alpha \operatorname{grad} u_{I} \cdot \operatorname{grad} \bar{v}_{I}+\operatorname{grad} u_{I} \cdot \overline{\mathbf{v}}_{\mathrm{II}}+\mathbf{u}_{\mathrm{II}} \cdot \operatorname{grad} \bar{v}_{\mathrm{I}}+u_{\mathrm{I}} \bar{v}_{\mathrm{I}}+2 \mathbf{u}_{\mathrm{II}} \cdot \overline{\mathbf{v}}_{\mathrm{II}}\right) d \Omega \\
& b(u, v)=\int_{\Omega}\left(u_{\mathrm{I}} \bar{v}_{\mathrm{I}}+\mathbf{u}_{\mathrm{II}} \cdot \overline{\mathbf{v}}_{\mathrm{II}}\right) d \Omega
\end{aligned}
$$

for $u=\left(u_{\mathrm{I}}, \mathbf{u}_{\mathrm{II}}\right), v=\left(v_{\mathrm{I}}, \mathbf{v}_{\mathrm{II}}\right) \in \mathbf{H}_{a}$ with $\alpha \in C^{\infty}(\bar{\Omega}), \alpha(x, y)>0$ and $(2 \alpha-1)(x, y)>0$ for $(x, y) \in \Omega$.

The inner product $a(\cdot, \cdot)$ gives a norm in $\mathbf{H}_{\mathfrak{a}}$ which is equivalent to the natural norm of this product space. By partial integration one shows that (2.1) is a weak form of the boundary value problem

$$
\begin{aligned}
-\operatorname{div}\left(\alpha \operatorname{grad} u_{\mathrm{I}}+\mathbf{u}_{\mathrm{II}}\right) & =(\lambda-1) u_{\mathrm{I}} & & \text { in } \quad \Omega \\
\operatorname{grad} u_{\mathrm{I}}+\mathbf{u}_{\mathrm{II}} & =(\lambda-1) \mathbf{u}_{\mathrm{II}} & & \text { in } \quad \Omega \\
u_{\mathrm{I}} & =0 & & \text { on } \partial \Omega .
\end{aligned}
$$

Here, the spectrum is non-negative and depends on the choice of $\alpha$. In any case, $\lambda=2$ is an eigenvalue of infinite multiplicity since for each $0 \neq u \in H^{2}(\Omega)$ an eigenpair of (2.1) is given by

$$
\left(2,\left(0,\left(u_{y},-u_{x}\right)\right)\right) \in \mathbb{R} \times \mathbf{H}_{a} .
$$

(a) In the Lehmann-Maehly approach (cf. Remark 2.2) we have $\mathbf{X}=\mathbf{H}_{a}, s(\cdot, \cdot)=$ $a(\cdot, \cdot)$ and $T=I$. Then, for each trial function $v_{i} \in \mathbf{H}_{a}$, the equation (2.4) which is a weak form of the following second order boundary value problem has to be solved for $w_{i}^{*}=\left(w_{i, \mathrm{I}}^{*}, \mathbf{w}_{i, \mathrm{II}}^{\star}\right) \in \mathbf{H}_{\mathbf{a}}$ :

$$
\begin{aligned}
-\operatorname{div}\left(\alpha \operatorname{grad} w_{i, \mathrm{I}}^{*}+\mathbf{w}_{i, \mathrm{II}}^{*}\right) & =v_{i, \mathrm{I}} & & \text { in } \Omega \\
\operatorname{grad} w_{i, \mathrm{I}}^{*}+\mathbf{w}_{i, \mathrm{II}}^{*} & =\mathbf{v}_{i, \mathrm{II}} & & \text { in } \Omega \\
w_{i, \mathrm{I}}^{*} & =0 & & \text { on } \partial \Omega .
\end{aligned}
$$

(b) In Goerisch's approach we set

$$
\begin{aligned}
\mathbf{X} & =L_{2}(\Omega) \times\left(L_{2}(\Omega)\right)^{2} \times\left(L_{2}(\Omega)\right)^{2} \\
s(u, v) & =\int_{\Omega}\left(\alpha \mathbf{u}_{I I I} \cdot \overline{\mathbf{v}}_{I I I}+\mathbf{u}_{\mathrm{III}} \cdot \overline{\mathbf{v}}_{\mathrm{II}}+\mathbf{u}_{\mathrm{II}} \cdot \overline{\mathbf{v}}_{\mathrm{III}}+u_{\mathrm{I}} \bar{v}_{\mathrm{I}}+2 \mathbf{u}_{\mathrm{II}} \cdot \overline{\mathbf{v}}_{\mathrm{II}}\right) d \Omega
\end{aligned}
$$


for

$$
u=\left(u_{\mathrm{I}}, \mathbf{u}_{\mathrm{II}}, \mathbf{u}_{\mathrm{III}}\right), v=\left(v_{\mathrm{I}}, \mathbf{v}_{\mathrm{II}}, \mathbf{v}_{\mathrm{III}}\right) \in \mathbf{X}
$$

and

$$
T: \mathbf{H}_{a} \ni\left(f_{\mathrm{I}}, \mathbf{f}_{\mathrm{II}}\right) \longmapsto\left(f_{\mathrm{I}}, \mathbf{f}_{\mathrm{II}}, \operatorname{grad} f_{\mathrm{I}}\right) \in \mathbf{X} .
$$

The linear equation (2.5) reduces to a weak form of a first order partial differential equation plus an algebraic equation for $w_{i}^{\star}=\left(w_{i, \mathrm{I}}^{\star}, \mathbf{w}_{i, \mathrm{II}}^{*}, \mathbf{w}_{i, \mathrm{IHI}}^{\star}\right) \in \mathbf{X}$ :

$$
\left.\begin{array}{rl}
-\operatorname{div}\left(\alpha \mathbf{w}_{i, \mathrm{III}}^{*}+\mathbf{w}_{i, \mathrm{II}}^{*}\right)+w_{i, \mathrm{I}}^{*} & =v_{i, \mathrm{I}} \\
\mathbf{w}_{i, \mathrm{III}}^{*}+2 \mathbf{w}_{i, \mathrm{II}}^{*} & =\mathbf{v}_{i, \mathrm{II}}
\end{array}\right\} \quad \text { in } \Omega .
$$

These are solved by

$$
w_{i}^{*}=\left(v_{i, \mathrm{I}}, \frac{\alpha}{2 \alpha-1} \mathbf{v}_{i, \mathrm{II}}, \frac{-1}{2 \alpha-1} \mathbf{v}_{i, \mathrm{II}}\right) \in \mathbf{X} .
$$

For each $\mathbf{w}_{j} \in H^{1}(\Omega) \times H^{1}(\Omega)$ admissible solutions of the homogeneous equation are given by

$$
w_{j}^{\circ}=\left(\operatorname{div}(2 \alpha-1) \mathbf{w}_{j},-\mathbf{w}_{j}, 2 \mathbf{w}_{j}\right) \in \mathbf{X}^{\circ} .
$$

In our calculations of bounds we restrict the problem to the symmetry classes (invariant subspace)

$$
\begin{aligned}
\mathbf{H}_{\boldsymbol{a}} & =H_{0}^{1}(\Omega)^{(0,0)} \times\left(L_{2}(\Omega)^{(1,0)} \times L_{2}(\Omega)^{(0,1)}\right) \\
\mathbf{X} & =L_{2}(\Omega)^{(0,0)} \times\left(L_{2}(\Omega)^{(1,0)} \times L_{2}(\Omega)^{(0,1)}\right) \times\left(L_{2}(\Omega)^{(1,0)} \times L_{2}(\Omega)^{(0,1)}\right),
\end{aligned}
$$

respectively. Here, $L_{2}(\Omega)^{\left(a_{1}, a_{2}\right)}$ denotes the subspace of $L_{2}(\Omega)$ formed of all functions symmetric with respect to the $i$-th axis, if $a_{i}=0$ and antisymmetric with respect to the same axis, if $a_{i}=1 \quad(i \in\{1,2\})$.

We use polynomial trial functions $v_{1}, \ldots, v_{n} \in \mathbf{H}_{a}$, orthogonalized with respect to the inner product $b(\cdot, \cdot)$ and generated via a suitable enumeration by the following functions in $\mathbf{H}_{\mathbf{a}}$ :

$$
\left.\begin{array}{l}
(x, y) \longmapsto\left(\left(x^{2}-1\right) x^{2 i}\left(y^{2}-1\right) y^{2 k},(0,0)\right) \\
(x, y) \longmapsto\left(0,\left(x^{2 i+1} y^{2 k}, 0\right)\right) \\
(x, y) \longmapsto\left(0,\left(0, x^{2 i} y^{2 k+1}\right)\right)
\end{array}\right\} \quad \text { for } i, k \in \mathbb{N}_{0}
$$

Furthermore, the solutions $w_{1}^{\circ}, \ldots, w_{m}^{\circ} \in \mathbf{X}^{\circ}$ of the homogeneous equation are constructed according to (3.2) with the following functions in $H^{1}(\Omega)^{(1,0)} \times H^{1}(\Omega)^{(0,1)}$ :

$$
(x, y) \longmapsto\left(x^{2 i+1} y^{2 k}, 0\right) \text { and }(x, y) \longmapsto\left(0, x^{2 i} y^{2 k+1}\right) \text {. for } i, k \in \mathbb{N}_{0} .
$$

Mathematica [23] was employed for the symbolic evaluation of all inner products and for the calculation of all eigenvalues with high precision. 
At first consider $\alpha(x, y)=1$ for $(x, y) \in \Omega$. The corresponding eigenvalue problem can be solved explicitly: A second eigenvalue of infinte multiplicity is given by $\lambda=1$ with eigenfunctions $(u,-\operatorname{grad} u) \in \mathbf{H}_{a}$ for every $0 \neq u \in H^{2}(\Omega) \cap H_{0}^{1}(\Omega)$. Let $\left(\mu_{k, l}, u_{k, l}\right) \in$ $I R \times H_{0}^{1}(\Omega)(k, l \in \mathbb{N})$ be the eigenpairs of the Laplacian operator $-\Delta u=\mu u$ with

$$
\begin{aligned}
\mu_{k, l} & =\left(k^{2}+l^{2}\right)\left(\frac{\pi}{2}\right)^{2} \\
u_{k, l}(x, y) & =\sin \left(\frac{k \pi}{2}(x+1)\right) \sin \left(\frac{l \pi}{2}(y+1)\right) \quad \text { for }(x, y) \in \Omega .
\end{aligned}
$$

Then the further eigenpairs of problem (2.1) are given by

$$
\left(2+\mu_{k, l},\left(\mu_{k, l} u_{k, l}, \operatorname{grad} u_{k, l}\right)\right) \in \mathbb{R} \times \mathbf{H}_{a} \quad(k, l \in \mathbb{N}) .
$$

As the Poincaré principle characterizes $\lambda=1$ only,

$$
1=\inf _{\substack{v \subset H_{a} \\ d i m v=i}} \max _{0 \neq v \in V} \frac{a(v, v)}{b(v, v)} \quad \text { for all } i \in \mathbb{N},
$$

the Rayleigh-Ritz approach does not give bounds to eigenvalues in $[2, \infty)$.

From the explicit knowledge of all eigenvalues $\lambda>2$, we obtain

$$
2<\lambda_{1}<22<\lambda_{2}=\lambda_{3}<40<\lambda_{4}<60<\lambda_{5}=\lambda_{6}<78<\lambda_{7}=\lambda_{8}<112<\lambda_{9}
$$

\begin{tabular}{|c|c|c|c|c|c|c|c|c|}
\hline$\rho$ & $\begin{array}{c}\lambda_{1}= \\
6.93480220\end{array}$ & $\begin{array}{c}\lambda_{2}= \\
26.6740110\end{array}$ & $\begin{array}{c}\lambda_{3}= \\
26.6740110\end{array}$ & $\begin{array}{c}\lambda_{4}= \\
46: 41322\end{array}$ & $\begin{array}{c}\lambda_{5}= \\
66.15243\end{array}$ & $\begin{array}{c}\lambda_{6}= \\
66.15243\end{array}$ & $\begin{array}{c}\lambda_{7}= \\
85.892\end{array}$ & $\begin{array}{c}\lambda_{8}= \\
85.892\end{array}$ \\
\hline 2 & $\begin{array}{c}\Lambda_{+1}^{\rho}= \\
6.93480220\end{array}$ & $\begin{array}{c}\Lambda_{+2}^{\rho}= \\
26.6740112\end{array}$ & $\begin{array}{c}\Lambda_{+3}^{\rho}= \\
26.6740112\end{array}$ & $\begin{array}{c}\Lambda_{+4}^{p}= \\
46.41562\end{array}$ & $\begin{array}{c}\Lambda_{+5}^{D}= \\
66.15690\end{array}$ & $\begin{array}{c}\Lambda_{+6}^{\rho}= \\
66.15690\end{array}$ & $\begin{array}{l}\Lambda_{+7}^{\rho}= \\
86.684\end{array}$ & \\
\hline 22 & $\begin{array}{c}\Lambda_{-1}^{\rho}= \\
6.93480220\end{array}$ & $\begin{array}{c}\Lambda_{+1}^{\rho}= \\
26.6740138\end{array}$ & $\begin{array}{c}\Lambda_{+2}^{\rho}= \\
26.6740138\end{array}$ & $\begin{array}{c}\Lambda_{+3}^{\rho}= \\
46.42534\end{array}$ & $\begin{array}{c}\Lambda_{+4}^{\rho}= \\
66.17674\end{array}$ & $\begin{array}{c}\Lambda_{+5}^{\nu}= \\
66.17679\end{array}$ & $\begin{array}{l}\Lambda_{+6}^{\rho}= \\
88.141\end{array}$ & $\begin{array}{l}\Lambda_{+7}^{\rho}= \\
88.424\end{array}$ \\
\hline 40 & $\begin{array}{c}\Lambda_{-3}^{\rho}= \\
6.93480220\end{array}$ & $\begin{array}{c}\Lambda_{-2}^{\rho}= \\
26.6740098\end{array}$ & $\begin{array}{c}\Lambda_{-1}^{\rho}= \\
26.6740098\end{array}$ & $\begin{array}{c}\Lambda_{+1}^{\rho}= \\
46.46094\end{array}$ & $\begin{array}{c}\Lambda_{+2}^{\rho}= \\
66.20349\end{array}$ & $\begin{array}{c}\Lambda_{+3}^{\rho}= \\
66.20366\end{array}$ & $\begin{array}{l}\Lambda_{+4}^{\rho}= \\
89.057\end{array}$ & $\begin{array}{l}\Lambda_{+5}^{\rho}= \\
89.361\end{array}$ \\
\hline 60 & $\begin{array}{c}\Lambda_{-4}^{\rho}= \\
6.93480220\end{array}$ & $\begin{array}{c}\Lambda_{-3}^{\rho}= \\
26.6740105\end{array}$ & $\begin{array}{c}\Lambda_{-2}^{\circ}= \\
26.6740105\end{array}$ & $\begin{array}{c}\Lambda_{-1}^{\rho}= \\
46.39284\end{array}$ & $\begin{array}{c}\Lambda_{+1}^{\rho}= \\
66.39106\end{array}$ & $\begin{array}{c}\Lambda_{+2}^{p}= \\
66.39200\end{array}$ & & $\begin{array}{l}\Lambda_{+4}^{\rho}= \\
91.340\end{array}$ \\
\hline 78 & $\begin{array}{c}\Lambda_{-6}^{\rho}= \\
6.93480220\end{array}$ & $\begin{array}{c}\Lambda_{-5}^{\rho}= \\
26.6740106\end{array}$ & $\begin{array}{c}\Lambda_{-4}^{\rho}= \\
26.6740106\end{array}$ & $\begin{array}{c}\Lambda_{-3}^{\rho}= \\
46.40672\end{array}$ & $\begin{array}{c}\Lambda_{-2}^{\rho}= \\
66.02755\end{array}$ & $\begin{array}{c}\Lambda_{-1}^{p}= \\
66.02806\end{array}$ & $\begin{array}{l}\Lambda_{+1}^{\rho}= \\
99.496\end{array}$ & $\begin{array}{l}\Lambda_{+2}^{\rho}= \\
99.667\end{array}$ \\
\hline 112 & $\begin{array}{c}\Lambda_{-8}^{\rho}= \\
6.93480220\end{array}$ & $\begin{array}{c}\Lambda_{-7}^{\rho}= \\
26.6740108\end{array}$ & $\begin{array}{c}\Lambda_{-6}^{\rho}= \\
26.6740108\end{array}$ & $\begin{array}{l}\Lambda_{-5}^{\rho}= \\
46.41070\end{array}$ & $\begin{array}{c}\Lambda_{-4}^{\rho}= \\
66.12991\end{array}$ & $\begin{array}{c}\Lambda_{-3}^{\rho}= \\
66.12996\end{array}$ & $\begin{array}{l}\Lambda_{-2}^{\rho}= \\
82.463\end{array}$ & $\begin{array}{l}\Lambda_{-1}^{\rho}= \\
82.881\end{array}$ \\
\hline
\end{tabular}

which allows to identify local notations for these eigenvalues with $\rho=2,22,40,60,78$, 112. Consequently by Theorem 2.4, the upper [lower] triangle indicated in Table 1 contains upper [lower] bounds to $\lambda_{1}, \ldots, \lambda_{8}$.

Table 1: $\Lambda_{+i}^{\rho}=\Lambda_{+i}^{\rho[72,56]}$ for $\rho=2$ and $\Lambda_{ \pm i}^{\rho}=\Lambda_{ \pm i}^{\rho[108,56]}$ for $\rho \neq 2 ; \alpha(x, y)=1$

We will continue our example setting $\alpha(x, y)=1+x^{2} y^{2}$ for $(x, y) \in \Omega$ at the end of the next section. 


\section{The Right-definite Goerisch method}

Let $\mathbf{H}_{b}$ be a separable, complex Hilbert space with inner product $b(\cdot, \cdot)$, and suppose that $a(\cdot, \cdot)$ is a Hermitian sesquilinear form in $\mathbf{H}_{b}$ with domain $\mathbf{H}_{a}$. Furthermore, $a(\cdot, \cdot)$ is assumed to be bounded from below and closed. We then consider the right-definite eigenvalue problem

$$
\left.\begin{array}{l}
\text { Seek } \lambda \in \mathbb{R} \text { and non-trivial } u \in \mathbf{H}_{\mathbf{a}} \text { such } \\
\text { that } a(u, v)=\lambda b(u, v) \text { holds for all } v \in \mathbf{H}_{\mathbf{a}} .
\end{array}\right\}
$$

Problem (4.1) is equivalent to the eigenvalue problem for the self-adjoint operator $A$ defined by

$$
\begin{aligned}
\mathcal{D}(A) & =\left\{u \in \mathbf{H}_{a}: a(u, v)=b(\hat{u}, v) \quad\left(v \in \mathbf{H}_{b}\right) \text { for some } \hat{u} \in \mathbf{H}_{b}\right\} \\
b(A u, v) & =a(u, v) \quad \text { for all } u \in \mathcal{D}(A), v \in \mathbf{H}_{a}
\end{aligned}
$$

(see, e.g., [13: Chapter VI, Section 2]). The operator $A$ is bounded from below. With

$$
\mathbf{H}=\mathbf{H}_{b} \quad \text { and } \quad\langle u, v\rangle=b(u, v) \text { for all } u, v \in \mathbf{H}_{b}
$$

the operator $A$ satisfies the assumptions of Section 1, where

$$
D_{\rho}=\left\{u \in \mathbf{H}_{a}: a(u, v)=\rho b(u, v) \text { for all } v \in \mathbf{H}_{a}\right\} \text {. }
$$

Again, we assume that $\mathbf{X}$ is a complex Hilbert space with inner product $s(\cdot, \cdot)$ and isometry $T: \mathbf{H}_{b} \longrightarrow \mathbf{X}$. For fixed $\rho \in \mathbb{R}$, application of Theorem 1.3 gives the following characterization for $\lambda_{-i}^{\rho}$.

Corollary 4.1: Set

$$
\mathbf{H}_{*}=\left\{v \in \mathbf{H}_{a}: a(v, u)=s(w, T u)\left(u \in \mathbf{H}_{\mathbf{a}}\right) \text { for some } w \in \mathbf{X}\right\} \text {. }
$$

Let $\rho^{-}<\rho$ and $1 \leq i \leq k_{\rho}^{-}$. Then $\mathbf{H}_{*}=\mathcal{D}(A)$ and

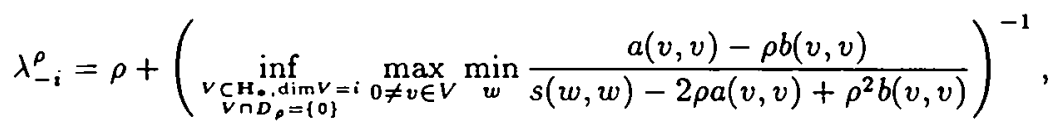

the minimum being taken over $w \in \mathbf{X}$ such that

$$
s(w, T u)=a(v, u) \quad \text { for all } u \in \mathbf{H}_{a} .
$$

Proof: Evidently, $\mathcal{D}(A) \subset \mathbf{H}_{*}$. To show the inclusion $\mathbf{H}_{*} \subset \mathcal{D}(A)$, suppose $v \in \mathbf{H}_{*}$ and let $w \in \mathbf{X}$ be such that condition (4.3) holds. Since $T \mathbf{H}_{b}$ is a closed subset of $X$ it possesses an orthogonal projector. Denote by $\widehat{w}$ the orthogonal projection of $w$ onto $T \mathbf{H}_{b}, \hat{w}=T \hat{u}$ for some $\hat{u} \in \mathbf{H}_{b}$. From $s(w-\hat{w}, T u)=0$ for all $u \in \mathbf{H}_{a} \subset \mathbf{H}_{b}$ then there follows

$$
a(v, u)=s(\hat{w}, T u)=b(\hat{u}, u) \quad \text { for all } u \in \mathbf{H}_{a}
$$

giving the inclusion $v \in \mathcal{D}(A)$. Assertion (4.2) is now an immediate consequence of Theorem 1.3, since the inclusion $w \in T A v+\left(T \mathbf{H}_{b}\right)^{\perp}$ is equivalent to the equality

$$
s(w, T u)=s(T A v, T u) \quad \text { for all } u \in \mathbf{H}_{b}=\overline{\mathbf{H}}_{a},
$$

and also to condition (4.3) 
We sketch briefly the discretization of (4.2), which is of course analogous to the discretization of (2.2). Suppose $n \in \mathbb{N}, m \subset \mathbb{N}_{0}$, and suppose the following:

(R1) $v_{1}, \ldots, v_{n} \in \mathcal{D}(A) \subset \mathbf{H}_{\mathbf{a}}$ are linearly independent.

(R2) $w_{1}^{\star}, \ldots, w_{n}^{\star} \in \mathbf{X}$ satisfy $s\left(w_{i}^{\star}, T u\right)=a\left(v_{i}, u\right)$ for all $u \in \mathbf{H}_{a} \quad(i=1, \ldots, n)$.

(R3) $w_{0}^{\circ}, \ldots, w_{m}^{\circ} \in \mathbf{X}^{\circ}$, where $w_{0}^{\circ}=0$ and $w_{1}^{\circ}, \ldots, w_{m}^{\circ}$ are linearly independent.

Again, the classical Lehmann-Maehly method is obtained by (1.8), choosing $\mathbf{X}=$ $\mathbf{H}_{b}, s(\cdot, \cdot)=b(\cdot, \cdot)$ and $T=I$. Set

$$
\begin{aligned}
& V_{n}=\operatorname{span}\left\{v_{1}, \ldots, v_{n}\right\}, \quad \mathbf{X}_{m}^{\circ}=\operatorname{span}\left\{w_{0}^{\circ}, \ldots, w_{m}^{\circ}\right\} \\
& a_{i k}^{(0)}=b\left(A v_{k}, A v_{i}\right), \quad a_{i k}^{(1)}=a\left(v_{k}, v_{i}\right) \quad a_{i k}^{(2)}=b\left(v_{k}, v_{i}\right) \quad(i, k=1, \ldots, n) \\
& b_{i k}^{(11)}=s\left(w_{k}^{*}, w_{i}^{*}\right) \quad(i, k=1, \ldots, n) \\
& b_{i k}^{(12)}=\left\{\begin{array}{ll}
s\left(w_{k}^{\circ}, w_{i}^{\star}\right) & \text { if } m>0 \\
0 & \text { if } m=0
\end{array} \quad(i=1, \ldots, n, k=1, \ldots, \max \{1, m\})\right. \\
& b_{i k}^{(22)}=\left\{\begin{array}{ll}
s\left(w_{k}^{\circ}, w_{i}^{\circ}\right) & \text { if } m>0 \\
1 & \text { if } m=0
\end{array} \quad(i, k=1, \ldots, \max \{1, m\})\right. \\
& A_{j}=\left(a_{i k}^{(j)}\right) \quad(j=0,1,2) \\
& B_{j l}=\left(b_{i k}^{(j l)}\right) \quad(j, l=1,2 ; j \leq l) .
\end{aligned}
$$

As in Section 2 we suppose $V_{n} \cap D_{\rho}=\{0\}$. Here, we have

$$
s\left(w^{\star}+w^{\circ}, w^{\star}+w^{\circ}\right)=x^{H}\left(B_{11}-B_{12} B_{22}^{-1} B_{12}^{H}\right) x \geq x^{H} A_{0} x
$$

for

$$
v=\sum_{i=1}^{n} x_{i} v_{i} \quad \text { with } x=\left(x_{1}, \ldots, x_{n}\right)^{t} \in \boldsymbol{C}^{n} \quad \text { and } \quad w^{\star}=\sum_{i=1}^{n} x_{i} w_{i}^{*}
$$

and $w^{\circ} \in \mathbf{X}_{m}^{\circ}$ defined by

$$
s\left(w^{\circ}, w\right)=-s\left(w^{\star}, w\right) \quad \text { for all } w \in \mathbf{X}_{m}^{\circ} .
$$

Consider the right-definite matrix eigenvalue problem

$$
\tau \in \mathbb{R}, x \in \boldsymbol{C}^{n}: \quad\left(A_{1}-\rho A_{2}\right) x=\tau\left(B_{11}-B_{12} B_{22}^{-1} B_{12}^{H}-2 \rho A_{1}+\rho^{2} A_{2}\right) x
$$

Denote its non-zero eigenvalues by $\tau_{i}^{-}$and $\tau_{i}^{+}$,

$$
\tau_{1}^{-} \leq \ldots \leq \tau_{n^{-}}^{-}<0<\tau_{n^{+}}^{+} \leq \ldots \leq \tau_{1}^{+}
$$

$n^{-}+n^{+} \leq n$, and set

$$
\begin{aligned}
& \Lambda_{-i}^{\rho[n, m]}=\rho+\left(\tau_{i}^{-}\right)^{-1} \text { for all } i=1, \ldots, n^{-} \\
& \Lambda_{+i}^{\rho[n, m]}=\rho+\left(\tau_{i}^{+}\right)^{-1} \text { for all } i=1, \ldots, n^{+} \text {. }
\end{aligned}
$$


Theorem 4.2: Let $\rho^{-}<\rho$ or $\rho<\rho^{+}$. Then, we have

$$
\begin{aligned}
& \Lambda_{-i}^{\rho[n, m]} \leq \lambda_{-i}^{\rho} \text { for } i=1, \ldots, \min \left\{n^{-}, k_{\rho}^{-}\right\} \\
& \Lambda_{+i}^{\rho[n, m]} \geq \lambda_{+i}^{\rho} \text { for } i=1, \ldots, \min \left\{n^{+}, k_{\rho}^{+}\right\},
\end{aligned}
$$

respectively.

Proof: We will prove the first inequality of (4.4) only. Here, one has

$$
\begin{aligned}
& \tau_{i}^{-}=\inf _{\substack{v \subset C^{n} \\
\operatorname{dim} V=i}} \max _{0 \neq v \in V} \frac{x^{H}\left(A_{1}-\rho A_{2}\right) x}{x^{H}\left(B_{11}-B_{12} B_{22}^{-1} B_{12}^{H}-2 \rho A_{1}+\rho^{2} A_{2}\right) x} \\
& =\inf _{\substack{v \subset V_{n} \\
\operatorname{dim} V=i}} \max _{0 \neq v \in V} \min _{w \in w^{*}+X_{m}^{\circ}} \frac{a(v, v)-\rho b(v, v)}{s(w, w)-2 \rho a(v, v)+\rho^{2} b(v, v)}
\end{aligned}
$$

with $V_{n} \subset \mathcal{D}(A)$ and $w^{\star}+\mathbf{X}_{m}^{\circ} \subset T A v+\mathbf{X}^{\circ}$. Theorem 1.3 yields

$$
\Lambda_{-i}^{\rho\{n, m\}}=\rho+\left(\tau_{i}^{-}\right)^{-1} \leq \rho+\left(\sigma_{i}^{-}\right)^{-1}=\lambda_{-i}^{\rho}
$$

and the assertion is proved

According to Theorem 4.2, the bound $\Lambda_{ \pm i}^{\rho[n, m]}$ is obtained through a shift and inversion process resembling Wielandt's inverse iteration. Here, only one step of the iteration is applied. Instead, the numbers $n, m$ are increased in order to achieve sufficient accuracy. In particular, the Lehmann-Maehly method for right-definite problems can be regarded as one step of Wielandt's inverse iteration for the subspace $V_{n} \subset \mathcal{D}(A)$. Consequently, Wielandt's eigenvalue approximations are in fact bounds to eigenvalues.

In order to compare the right- and left-definite methods, assume that problem (4.1) satisfies also the assumptions of Section 2. Then, both versions can be applied, each of them involving the Rayleigh-Ritz matrices $A_{1}=\left(a\left(v_{k}, v_{i}\right)\right)_{i k}$ and $A_{2}=\left(b\left(v_{k}, v_{i}\right)\right)_{i k}$. In addition,

$$
\begin{aligned}
& B_{11}-B_{12} B_{22}^{-1} B_{12}^{H} \simeq A_{0}=\left(b\left(A v_{k}, A v_{i}\right)\right)_{i k} \\
& C_{11}-C_{12} C_{22}^{-1} C_{12}^{H} \simeq A_{3}=\left(a\left(B v_{k}, B v_{i}\right)\right)_{i k}
\end{aligned}
$$

are required in the respective approaches, $B=A^{-1}$.

Consider e.g. the eigenvalue problem given in Section 3. With $v_{i} \in \mathcal{D}(A)$ the elements $A v_{i}$ can be obtained through differentiation,

$$
A v_{i}=\left(-\operatorname{div}\left(\alpha \operatorname{grad} v_{i, \mathrm{I}}+\mathbf{v}_{i, \mathrm{II}}\right)+v_{i, \mathrm{I}}, \operatorname{grad} v_{i, \mathrm{I}}+2 \mathbf{v}_{i, \mathrm{II}}\right)
$$

whereas $B v_{i}$ is the (weak) solution of a boundary value problem, namely (3.1). When applying the right-definite version difficulties mostly arise in finding trial functions $v_{i}$ which are sufficiently smooth and fulfill all boundary conditions.

Continuing the numerical example of Section 3 , we set $\alpha(x, y)=1+x^{2} y^{2}$ for $(x, y) \epsilon$ $\Omega$. In this case, there exists essential spectrum $\sigma_{e}=\left\{1, \frac{3}{2}\right\} \cup\{2\}$ and point spectrum in $(2,+\infty)$. Denote the $i$-th eigenvalue above 2 by $\lambda_{i}$. Since the operator $A$ in $\mathbf{H}$ is well known the right-definite Lehmann-Maehly method can be applied to the trial functions 
$v_{i} \in \mathcal{D}(A)$ of Section 3 and the elements given in (4.5). Again, $\rho=2=\max \sigma_{\mathrm{e}}$ provides upper bounds to $\lambda_{i}$. We then successively choose $\rho \approx 0.9 \Lambda_{+i}^{2[56,0]}$ for $i=2,4,5,7,9$. Under the heuristic assumption that

$$
2<\lambda_{1}<25<\lambda_{2} \leq \lambda_{3}<46<\lambda_{4}<62<\lambda_{5} \leq \lambda_{6}<82<\lambda_{7} \leq \lambda_{8}<117<\lambda_{9}
$$

Theorem 4.2 gives upper [lower] bounds to $\lambda_{1}, \ldots, \lambda_{8}$ shown in the upper [lower] triangle of Table 2.

\begin{tabular}{|c|c|c|c|c|c|c|c|c|}
\hline$\rho$ & $\lambda_{1}$ & $\lambda_{2}$ & $\lambda_{3}$ & $\lambda_{4}$ & $\lambda_{5}$ & $\lambda_{6}$ & $\lambda_{7}$ & $\lambda_{8}$ \\
\hline 2 & $\begin{array}{c}\Lambda_{+1}^{\rho}= \\
7.206691786\end{array}$ & $\begin{array}{c}\Lambda_{+2}^{\rho}= \\
27.473166\end{array}$ & $\begin{array}{c}\Lambda_{+3}^{\rho}= \\
28.305436\end{array}$ & $\begin{array}{c}\Lambda_{+4}^{\rho}= \\
50.5920\end{array}$ & $\begin{array}{l}\Lambda_{+5}^{\rho}= \\
68.6623\end{array}$ & $\begin{array}{l}\Lambda_{+6}^{\rho}= \\
68.7772\end{array}$ & $\begin{array}{l}\Lambda_{+7}^{\rho}= \\
91.718\end{array}$ & $\begin{array}{l}\Lambda_{+8}^{\rho}= \\
96.518\end{array}$ \\
\hline 25 & $\begin{array}{c}\Lambda_{-1}^{\rho}= \\
7.206691735\end{array}$ & $\begin{array}{c}\Lambda_{+1}^{\rho}= \\
27.473373\end{array}$ & $\begin{array}{c}\Lambda_{+2}^{\rho}= \\
28.305513\end{array}$ & $\begin{array}{c}\Lambda_{+3}^{\rho}= \\
50.5943\end{array}$ & $\begin{array}{l}\Lambda_{+4}^{\rho}= \\
68.6639\end{array}$ & $\begin{array}{c}\Lambda_{+5}^{p}= \\
68.7786\end{array}$ & $\begin{array}{l}\Lambda_{+6}^{\nu}= \\
91.811\end{array}$ & $\begin{array}{l}\Lambda_{+7}^{\rho}= \\
96.699\end{array}$ \\
\hline 46 & $\begin{array}{c}\Lambda_{-3}^{\rho}= \\
7.206691744\end{array}$ & $\begin{array}{c}\Lambda_{-2}^{\rho}= \\
27.473124\end{array}$ & $\begin{array}{c}\Lambda_{-1}^{\rho}= \\
28.305411\end{array}$ & $\begin{array}{c}\Lambda_{+1}^{\rho}= \\
50.6110\end{array}$ & $\begin{array}{l}\Lambda_{+2}^{\rho}= \\
68.6672\end{array}$ & $\begin{array}{c}\Lambda_{+3}^{\rho}= \\
68.7814\end{array}$ & $\begin{array}{l}\Lambda_{+4}^{\rho}= \\
91.896\end{array}$ & $\begin{array}{l}\Lambda_{+5}^{\rho}= \\
96.896\end{array}$ \\
\hline 62 & $\begin{array}{c}\Lambda_{-4}^{\rho}= \\
7.206691747\end{array}$ & $\begin{array}{c}\Lambda_{-3}^{\rho}= \\
27.473138\end{array}$ & $\begin{array}{c}\Lambda_{-2}^{p}= \\
28.305419\end{array}$ & $\begin{array}{c}\Lambda_{-1}^{\rho}= \\
50.5824\end{array}$ & $\begin{array}{c}\Lambda_{+1}^{\rho}= \\
68.6833\end{array}$ & $\begin{array}{c}\Lambda_{+2}^{\rho}= \\
68.7949\end{array}$ & $\begin{array}{l}\Lambda_{+3}^{\rho}= \\
92.036\end{array}$ & $\begin{array}{l}\Lambda_{+4}^{\rho}= \\
97.186\end{array}$ \\
\hline 82 & $\begin{array}{c}\Lambda_{-6}^{\rho}= \\
7.206691749\end{array}$ & $\begin{array}{c}\Lambda_{-5}^{\rho}= \\
27.473144\end{array}$ & $\begin{array}{c}\Lambda_{-4}^{p}= \\
28.305422\end{array}$ & $\begin{array}{c}\Lambda_{-3}^{\rho}= \\
50.5877\end{array}$ & $\begin{array}{c}\Lambda_{-2}^{\rho}= \\
68.6493\end{array}$ & $\begin{array}{c}\Lambda_{-1}^{\rho}= \\
68.7658\end{array}$ & $\begin{array}{l}\Lambda_{+1}^{p}= \\
92.844\end{array}$ & $\begin{array}{l}\Lambda_{+2}^{\rho}= \\
98.844\end{array}$ \\
\hline 117 & $\begin{array}{c}\Lambda_{-8}^{\rho}= \\
7.206691750\end{array}$ & $\begin{array}{c}\Lambda_{-7}^{\rho}= \\
27.473148\end{array}$ & $\begin{array}{c}\Lambda_{-6}^{\rho}= \\
28.305424\end{array}$ & $\begin{array}{c}\Lambda_{-5}^{\rho}= \\
50.5894\end{array}$ & $\begin{array}{l}\Lambda_{-4}^{\rho}= \\
68.6573\end{array}$ & $\begin{array}{c}\Lambda_{-3}^{\rho}= \\
68.7730\end{array}$ & $\begin{array}{l}\overline{\Lambda_{-2}^{\rho}}= \\
91.215\end{array}$ & $\begin{array}{l}\overline{\Lambda_{-1}^{\rho}}= \\
94.973\end{array}$ \\
\hline
\end{tabular}

Table 2: $\quad \Lambda_{+i}^{\rho}=\Lambda_{+i}^{\rho[58,0]}$ for $\rho=2$ and $\Lambda_{ \pm i}^{\rho}=\Lambda_{ \pm i}^{\rho[84,0]}$ for $\rho \neq 2 ; \alpha(x, y)=1+x^{2} y^{2} ;$ $\Lambda_{+9}^{2[56,0]}=130.32$.

Acknowledgement. The authors would like to thank Professor Waldemar Velte whose comments helped to improve the presentation of this paper.

\section{References}

[1] Alama, S., Deift, P. A. and R. Hempel: Eigenvalue branches of the Schrödinger operator $H-\lambda W$ in a gap of $\sigma(W)$. Comm. Math. Phys. 121 (1989), $291-321$.

[2] Albrecht, J.: Einschließung von Eigenwerten bei Schwingungen von Kreisbögen. Z. Angew. Math. Mech. (ZAMM) 63 (1983), 387 - 389.

[3] Albrecht, J. and F. Goerisch: Anwendungen des Verfahrens von Lehmann auf Schwingungsprobleme. In: Numerical Treatment of Eigenvalue Problems (Vol. 4) (Intern. Ser. Numer. Math.: Vol. 83) (eds: J. Albrecht et al). Basel-Boston: Birkhäuser Verlag 1987, pp. 1 - 9.

[4] Behnke, H.: Calculation of bounds for sloshing frequencies with the use of finite elements. Z. Angew. Math. Mech. (ZAMM) 67 (1987), T 347 - T 349.

[5] Behnke, H. and F. Goerisch: Inclusions for eigenvalues of selfadjoint problems. In: Topics in Validated Computations (ed.: J. Herzberger). Amsterdam: Elsevier (North-Holland) 1994, pp. $277 \cdot 322$. 
[6] Behnke, H. and U. Mertins: Eigenwertschranken für das Problem der freischwingenden rechteckigen Platte und Untersuchungen zum Ausweichphänomen. Z. Angew. Math. Mech. (ZAMM) 75 (to appear).

[7] Descloux, J., Nassif, N. and J. Rappaz: On spectral approximation. Parts 1 and 2. RAIRO Anal. Numér. 12 (1978), $97-112$ and $113-119$.

[8] Goerisch, F.: Eine Verallgemeinerung eines Verfahrens von N. J. Lehmann zur Einschließung von Eigenwerten. Wiss. Z. Tech. Univ. Dres. 29 (1980), $429-431$.

[9] Goerisch, F.: Eigenwertschranken und komplementäre Extremalprinzipien. Habilitationsschrift. Clausthal: Techn. Univ. 1986.

[10] Goerisch, F. and H. Haunhorst: Eigenwertschranken für Eigenwertaufgaben mit partiellen Differentialgleichungen. Z. Angew. Math. Mech. (ZAMM) 65 (1985), 129 - 135.

[11] Goerisch, F. and S. Zimmermann: On Trefft's method and its application to eigenvalue problems. Z. Angew. Math. Mech. (ZAMM) 66 (1986), T 304 - T 306.

[12] Gruber, R. and J. Rappaz: Finite Element Methods in Linear Ideal Magnetohydrodynamics. Berlin - Heidelberg: Springer-Verlag 1985.

[13] Kato,T.: Perturbation Theory for Linear Operators (2nd ed.). Berlin - Heidelberg - New York: Springer-Verlag 1976.

[14] Lehmann, N. J.: Beiträge zur numerischen Lösung linearer Eigenwertprobleme. Parts I and II. Z. Angew. Math. Mech. (ZAMM) 29 (1949), $341-356$ and 30 (1950), 1 - 16.

[15] Maehly, H. J.: Ein neues Verfahren zur genäherten Berechnung der Eigenwerte hermitescher Operatoren. Helv. Phys. Acta 25 (1952), 547 - 568.

[16] Messiah, A.: Quantum Mechanics. Vol. 2. Amsterdam: North-Holland. 1970.

[17] Raikov, G. D.: The spectrum of a linear magnetohydrodynamic model with cylindrical symmetry. Arch. Rat. Mech. Anal. 116 (1991), 161 - 198.

[18] Rappaz, J.: Approximation of the spectrum of a non-compact operator given by the magnetohydrodynamic stability of plasma. Numer. Math. 28 (1977), 15 - 24.

[19] Velte,W.: Direkte Methoden der Variationsrechnung. Stuttgart: B. G. Teubner 1976.

[20] Weidmann, J.: Oszillationsmethoden für Systeme gewöhnlicher Differentialgleichungen. Math. Z. 119 (1971), 349 - 373.

[21] Weinberger, H. F.: Variational Methods for Eigenvalue Approximation. Philadelphia (Pennsylvania): SIAM 1974.

[22] Weinstein, A. and W. Stenger: Methods of Intermediate Problems for Eigenvalues. New York - London: Acad. Press 1972.

[23] Wolfram, S.: Mathematica. A System for Doing Mathematics by Computer (2nd ed.). Redwood City (California): Addison-Wesley 1991.

[24] Zimmermann, S.: Comparison of errors in upper and lower bounds to eigenvalues of selfadjoint eigenvalue problems. Numer. Funct. Anal. Optim. 15 (1994), 943 - 960. 